\title{
Convergência e Mudanças Tecnológicas: uma Análise das Emissões de Gases de Efeito Estufa
}

\section{Convergence and Technological Change: an Analysis of Emissions of Greenhouse Gases}

\author{
Lucas Vitor de Carvalho Sousa* \\ Samira Martins Guimarães** \\ Adriano Provezano Gomes* * *
}

\begin{abstract}
Resumo: As emissões de gases de efeito estufa contribuem para o aquecimento global, o que torna necessária a adoção de medidas para a mitigação desses gases, como o progresso tecnológico e maior eficiência produtiva. Dessa forma, o presente trabalho busca verificar se os países do Anexo I e do Não Anexo I do Protocolo de Quioto obtiveram mudanças tecnológicas e de eficiência, bem como uma possível convergência da eficiência entre os países no período de 1995 a 2010. Para isso, utiliza-se a análise envoltória de dados, que, por meio do índice de Malmquist, permite verificar essas mudanças. Os resultados mostram que houve mudança tecnológica e convergência na eficiência, o que demonstra maior preocupação dos países com relação às mudanças climáticas.
\end{abstract}

Palavras-chave: Emissões de gases de efeito estufa. Eficiência. Convergência na eficiência.

\begin{abstract}
Emissions of greenhouse gases contribute to global warming, which makes it necessary to adopt measures for the mitigation of greenhouse gases, such as technological progress and productive efficiency. Thus, this paper seeks to ascertain whether the countries of Annex I and Non-Annex I of the Kyoto Protocol achieved technological and efficiency changes, as well as a possible convergence of efficiency across countries for the period 1995-2010. For this, we used the Data Envelopment Analysis, which by means of the Malmquist Index can verify these changes. The results showed technological change and convergence efficiency, demonstrating the greater concern of countries in relation to climate change.
\end{abstract}

Keywords: Emissions of greenhouse gases. Efficiency. Convergence efficiency.

JEL Classification: O3; Q54.

\footnotetext{
* Doutorando em Economia pela Universidade de Brasília (UnB).E-mail: lucasvitor.cs@gmail.com

** $\quad$ Mestre em Economia pela Universidade Federal de Viçosa (UFV). E-mail: guimaraesmsamira@ gmail.com

*** Doutor em Economia Aplicada pela Universidade Federal de Viçosa (UFV). Professor do Programa de Pós-Graduação em Economia da Universidade Federal de Viçosa (UFV). E-mail: apgomes@ufv.br
} 


\section{1 lntrodução}

A relação entre bem-estar e meio ambiente é um tema controverso e de grande relevância para as economias atuais, ainda mais em um cenário de aquecimento global. O mais recente relatório do Painel Intergovernamental sobre Mudança do Clima (INTERGOVERNMENTAL PANEL ON CLIMATE CHANGE, 2013) afirma que o aquecimento do sistema climático é inequívoco e desde o período pré-industrial a concentração de gás carbônico na atmosfera aumentou 40\%. Evitar o aumento desastroso da temperatura exigirá a redução e a estabilização dos níveis de emissões de gases de efeito estufa (GEE). Entre os fatores antrópicos, a queima de combustíveis fósseis é a principal causadora das emissões, o que leva a um trade-off entre preservar o meio ambiente e manter o crescimento econômico. Ambos são fundamentais para o bem-estar, e priorizar algum em sacrifício do outro não parece ser a melhor alternativa. A saída para esse grande problema pode ser encontrada no progresso tecnológico e na eficiência produtiva em âmbito global.

Alguns trabalhos empíricos argumentam que as políticas climáticas afetariam negativamente o crescimento econômico e a competitividade (BRÄNNLUND; LUNDGREN, 2009; FEIJÓ; AZEVEDO, 2006; JAFFE et al. 1995). Outros defendem que as mudanças tecnológicas reduziriam os custos de mitigação, tornando viáveis as políticas climáticas (DE LA TORRE; FAJNZYLBER; NASH, 2008; FISCHER; NEWELL, 2008; INTERNATIONAL ENERGY ADMINISTRATION, 2008; MILLS, 2009). Como destacam Endehofer, Bauer e Kruegler (2005), o potencial da mudança tecnológica na redução dos custos de mitigação é um pré-requisito para o desenvolvimento de uma política climática. Devido a essas evidências, o presente artigo tem como objetivo verificar se os países vinculados ao Protocolo de Quioto (países do Anexo I ${ }^{1}$ e do Não Anexo I) obtiveram mudanças tecnológicas que mitiguem as emissões de GEE, o que deveria ocorrer entre 2008 e 2012. Especificamente, o presente estudo procurou analisar se há convergência na eficiência dos países analisados quando se consideram as emissões de GEE. Para isso, é utilizada a análise envoltória de dados (DEA) (sigla em inglês de data envelopment analysis), que, através do índice de Malmquist, permite verificar mudanças tecnológicas e de eficiência.

O Protocolo de Quioto, realizado em 1997, no Japão, estabeleceu que os países industrializados (países do Anexo I) deveriam reduzir as emissões de GEE

$1 \quad$ De acordo com a United Nations (2013), os países que fazem parte do Anexo I são: Alemanha, Austrália, Áustria, Bélgica, Bielorússia, Bulgária, Canadá, Croácia, Chipre, República Checa, Dinamarca, Eslováquia, Eslovênia, Espanha, Estados Unidos, Estônia, Finlândia, França, Grécia, Hungria, Islândia, Irlanda, Itália, Japão, Letônia, Liechtenstein, Lituânia, Luxemburgo, Malta, Mônaco, Holanda, Nova Zelândia, Noruega, Polônia, Portugal, Reino Unido, Romênia, Rússia, Suécia, Suíça, Turquia, Ucrânia. 
em pelo menos 5\% em relação aos níveis de 1990 até o período entre 2008 e 2012. Tal compromisso é uma tentativa de reversão da tendência global de aumento das emissões ocorridas desde a revolução industrial. O Protocolo foi aberto para assinatura em 16 de março de 1998 e entraria em vigor 90 dias após a ratificação de pelo menos 55 partes (países), que juntas produzissem 55\% das emissões globais, o que ocorreu somente em 2005 com a ratificação da Rússia em novembro de 2004 (BRASIL, 1997). Embora os países do Não Anexo I não tivessem obrigações formais quanto à mitigação das emissões de GEE, alguns assumiram, voluntariamente, o compromisso de reduzir suas emissões. Evidentemente, atingir a meta de emissões proposta pelo Protocolo de Quioto exige transformações no processo produtivo das nações, o que implica em mudanças tecnológicas e de eficiência produtiva.

Apesar das grandes conferências realizadas nos últimos anos e uma maior preocupação a respeito das mudanças climáticas, o fato de ainda haver grande discrepância quanto à mitigação de GEE entre os países leva ao questionamento da hipótese de convergência. Em outras palavras, os países menos eficientes na formação do PIB estariam se desenvolvendo de forma sustentável mais rapidamente, quando se consideram as emissões de GEE, e, dessa forma, alcançando os mais eficientes? Caso seja confirmada essa hipótese, esses países se beneficiariam dos ganhos de eficiência, o que significa, no longo prazo, menor diferença entre países com baixas e altas eficiências, como também menores impactos no sistema climático.

Este artigo apresenta cinco seções, incluindo esta introdução. A seção 2 faz uma breve revisão teórica a respeito do crescimento econômico e as questões ambientais. A seção 3 apresenta os procedimentos metodológicos utilizados no trabalho, elucidando os métodos empregados, a seleção de variáveis e a especificação dos modelos. A seção 4 traz a análise dos resultados. Por fim, a seção 5 traça as considerações finais.

\section{Crescimento Econômico, Tecnologia e Questões Ambientais}

Solow (1956) foi um dos pioneiros a desenvolver um modelo de crescimento econômico embasado principalmente no capital físico. Diferentemente dos modelos de Harrod (1939) e Domar (1946), em que o equilíbrio se encontra sobre o "fio da navalha" (equilíbrio instável), Solow (1956) formulou um modelo de crescimento em que se pressupõe substitubilidade entre os fatores de produção. Dessa forma, a hipótese de equilíbrio instável pode ser descartada. Entretanto, como o insumo capital que sustentava o crescimento apresentava retornos decrescentes, o único modo de manter o crescimento era por meio de uma variável exógena: o 
progresso tecnológico. Assim, mantendo as demais variáveis constantes, somente um choque tecnológico exógeno promoveria o crescimento econômico.

No modelo de Solow $(1956,1957)$ existem quatro variáveis que compõem a função de produção Cobb-Douglas: o trabalho $(L)$, o capital $(K)$, a tecnologia $(A)$ e o produto (Y). O progresso técnico ocorre quando A aumenta ao longo do tempo, deixando o trabalho mais produtivo, por exemplo. Dessa forma, o progresso tecnológico é a variável chave que gera o crescimento econômico.

Entretanto, como haver capital sem recursos naturais? E, por conseguinte, como manter o crescimento econômico? De fato, como destaca Romer (2006), os recursos naturais, a poluição e outras considerações acerca do meio ambiente estiveram ausentes da maioria dos estudos em economia. Solow, por exemplo, deu destaque para os recursos naturais somente em 1974 e em 1986 (SOLOW, 1974, 1986), porém mantendo a função de produção Cobb-Douglas, que pressupõe substitubilidade entre os insumos, incluindo os recursos naturais.

Segundo Solow (1986), a economia é grande o suficiente para que os ganhos de especialização dos fatores fossem exauridos, e no caso de ocorrer uma restrição relativa, esta poderia ser superada pelo progresso tecnológico. Dessa forma, se o avanço tecnológico for maior que a taxa de consumo do capital natural, o crescimento econômico pode ser sustentável. Mas isso só é possível graças à característica de substitubilidade da função de produção Cobb-Douglas, que, mesmo ao considerar a finitude dos recursos naturais, não a vê como uma restrição, pois uma alteração positiva no progresso tecnológico modifica a produção independentemente do valor da taxa de desgaste dos recursos naturais (ROMER, 2006). Essa visão é conhecida na literatura como sustentabilidade fraca, ou seja, a escassez de recursos naturais não representa uma restrição ao crescimento econômico, pois podem ser substituídos por capital manufaturado (NEUMAYER, 2003).

Pode-se perceber que o processo de desenvolvimento de novas tecnologias, ou seja, o progresso tecnológico, é o fator chave para o crescimento econômico em modelos neoclássicos. Porém, muitos desses modelos estão fundamentados nas propriedades da função de produção Cobb-Douglas, que pressupõe substitubilidade entre os insumos. Autores como Georgescu-Roegen (1986), Daly (1997), Daly e Farley (2004), entre outros, enxergam a economia como um subsistema aberto de um todo maior, que a sustenta e a contém. Esse sistema maior, como esclarecem Daly e Farley (2004), é considerado finito, não aumenta e é materialmente fechado, apesar de aberto à energia solar. Essa abordagem defende que 
recursos naturais e capital manufaturado são essencialmente complementares e não podem substituir um ao outro, sendo então conhecida como sustentabilidade forte (NEUMAYER, 2003).

Nesse contexto, o progresso científico e tecnológico é essencial para aumentar a eficiência no uso dos recursos naturais em geral (renováveis e não renováveis), mas não é suficiente para substituí-los. A sustentabilidade da economia, no longo prazo, não é possível sem a estabilização dos níveis de consumo per capita de acordo com a capacidade de suporte do planeta. A partir dessa constatação, uma função de produção na forma multiplicativa como a de Cobb-Douglas pode não representar com fidelidade a realidade, uma vez que a hipótese de substitubilidade entre insumos naturais e não naturais é um tema controverso.

Na maioria dos estudos econômicos, os teóricos aceitam os pressupostos da função Cobb-Douglas, inclusive o de substitubilidade entre os insumos produtivos, pela sua simplificação e facilidade matemática e analítica. Contudo, as ideias formadas a partir dessa função, apesar de atraentes, levam a conclusões limitadas. Uma alternativa à função de produção Cobb-Douglas é a utilização de modelos não paramétricos como a DEA. Esse método é uma análise comparativa entre unidades tomadoras de decisão (DMU) (sigla em inglês de decision making unit), que neste estudo são os países do Anexo I e do Não Anexo I do Protocolo de Quioto. A DMU que estiver sobre a fronteira de possibilidades de produção é a mais eficiente em utilizar seus insumos para produzir seus produtos. Embora a metodologia DEA permita a substitubilidade entre os insumos, a fronteira de possibilidades de produção se ajusta à disposição dos dados, permitindo maior comparabilidade entre os países em estudo, sem a necessidade de uma forma funcional pré-estabelecida (FERREIRA; GOMES, 2012).

\section{Metodologia}

\subsection{Análise Envoltória de Dados (DEA)}

A DEA tem como finalidade medir a eficiência de um conjunto de DMU (empresas, departamentos, cidades, países, etc.) que consomem múltiplos insumos (inputs) para produzir múltiplos produtos (outputs) (SILVEIRA; MEZA; MELLO, 2012), sendo que uma de suas vantagens é a não imposição de formas funcionais para a fronteira de possibilidades de produção, além de ser uma técnica comparativa. Outra vantagem é que, como os inputs e outputs não necessitam estabelecer relações funcionais, isso possibilita que os insumos e produtos possuam unidades de medida diferentes.

A primeira modelagem de DEA foi proposta por Charnes et al. (1978), e a principal ideia dessa modelagem é encontrar a melhor DMU virtual para cada 
DMU real. Ou seja, pretende-se encontrar a DMU virtual, que consegue produzir maiores quantidades de produtos utilizando a mesma ou uma menor quantidade de insumos, então a DMU real com essas características será eficiente.

De acordo com Coelli et al. (2005), existem dois modelos clássicos de DEA: o modelo de retornos constantes à escala, que foi proposto por Charnes, Cooper $\mathrm{e}$ Rhodes, em 1978, conhecido também por CCR, em homenagem aos autores; e o modelo de retornos variáveis à escala, proposto por Banker, Charnes e Cooper, em 1984, também conhecido como BCC, que assume a não proporcionalidade entre os inputs e outputs e admite a decomposição da eficiência técnica em eficiência pura e eficiência de escala.

Os modelos DEA podem ser orientados a insumos ou a produtos. Nos modelos orientados a insumos, admite-se que a produção permaneça constante e que os insumos variem para que a DMU alcance a fronteira eficiente. Por outro lado, na modelagem orientada a produto, são os insumos que permanecem fixos, e a produção varia para alcançar a fronteira eficiente (FERREIRA; GOMES, 2012).

Segundo Charnes et al. (1994) e Lins e Meza (2000), os modelos de DEA com orientação a produto e pressuposição de retornos constantes à escala podem ser representados algebricamente:

$$
\begin{gathered}
\operatorname{MAX}_{\theta, \lambda} \varphi \\
\text { sujeito a: } \\
-\varphi \mathrm{y}_{\mathrm{i}}+\mathrm{Y} \lambda \geq 0, \\
\mathrm{X}_{\mathrm{i}} \mathrm{X} \lambda \geq 0, \\
\lambda \geq 0
\end{gathered}
$$

em que: $y_{i}$ é um vetor $(m \times 1)$ de quantidades de produto da i-ésima DMU; $x_{i}$ é um vetor ( $k \times 1)$ de quantidades de insumo da i-ésima DMU; Y é a matriz $(n \times \mathrm{m})$ de produtos das $n$ DMU; $X$ é uma matriz ( $n \times \mathrm{k}$ ) de insumos das $n$ DMU; $\lambda$ é um vetor $(n \times 1)$ de pesos; $\theta$ é eficiência técnica que varia entre 0 e 1 ; e $\varphi$ é o aumento proporcional nos produtos que poderiam ser obtidos pela i-ésima DMU, mantendo-se constante a utilização dos insumos.

O problema de programação linear (PPL) apresentado em 1 é resolvido $n$ vezes, uma vez para cada DMU, e apresenta os valores de $\theta$ e $\lambda$ como solução. Caso a DMU seja ineficiente, os valores de $\lambda$ fornecem os "pares" daquela unidade, ou seja, apresentam as DMU eficientes que servirão para aquela que foi ineficiente.

Banker et al. (1984) incorporaram a possibilidade de retornos variáveis à escala ao modelo de DEA ao introduzirem uma restrição de convexidade no modelo de retornos constantes à escala. A pressuposição de retornos variáveis à escala permite decompor a eficiência técnica em eficiência de escala e pura eficiência 
técnica. Dessa forma, o modelo de DEA com orientação a produto e com pressuposição de retornos variáveis à escala pode ser apresentado da seguinte forma:

$$
\begin{gathered}
\mathrm{MAX} \theta, \lambda \varphi \\
\text { sujeito a: } \\
-\varphi \mathrm{y}_{\mathrm{i}}+\mathrm{Y} \lambda \geq 0, \\
\mathrm{X}_{\mathrm{i}}-\mathrm{X} \lambda \geq 0, \\
\mathrm{~N}^{\prime} \lambda=1 \\
\lambda \geq 0
\end{gathered}
$$

em que N1 é um vetor ( $n \times 1)$ de números uns. As demais variáveis têm a mesma interpretação das descritas anteriormente. Esse enfoque forma uma superfície côncava de vários planos de interseção, cuja superfície envolve os dados de forma mais compacta do que a superfície formada pelos retornos constantes. Dessa forma, os valores obtidos para a eficiência técnica, com a pressuposição de retornos variáveis, são maiores ou iguais aos obtidos com retornos constantes à escala.

\subsection{0 Índice de Malmquist}

Para se analisar a mudança na eficiência técnica ao longo do tempo faz-se necessário utilizar um modelo que permita a visualização da dinâmica das DMU ao longo do período de análise. Dessa forma, propõe-se o índice de Malmquist, que permite a análise de mudanças na produtividade total de fatores (PTF) utilizando a metodologia de DEA.

O índice de Malmquist foi originalmente proposto por Caves et al. (1982), baseado no trabalho de Sten Malmquist em 1953, que teve como objetivo a elaboração de um índice de quantidade para a análise do consumo em razão de funções de distância. Segundo Ferreira e Gomes (2012), o índice de Malmquist pode ser definido pela função distância, sendo utilizada para agrupar a natureza multiproduto e multi-insumo, utilizando-se a DEA.

Segundo Färe et al. (1994), o índice de Malmquist orientado a produto, Mo, visa analisar as mudanças na produtividade total de fatores caracterizados por uma tecnologia de produção que objetiva a maximização proporcional do vetor produto, entre o período $t$ e $t+1$, podendo ser representado da seguinte forma:

$$
M_{O}\left(y_{t}, x_{t}, y_{t+1}, x_{t+1}\right)=\frac{d_{O}^{t+1}\left(y_{t+1}, x_{t+1}\right)}{d_{O}^{t}\left(y_{t}, x_{t}\right)}\left[\frac{d_{o}^{t}\left(y_{t+1}, x_{t+1}\right)}{d_{O}^{t+1}\left(y_{t+1}, x_{t+1}\right)} \times \frac{d_{o}^{t}\left(y_{t}, x_{t}\right)}{d_{O}^{t+1}\left(y_{t}, x_{t}\right)}\right]^{1 / 2}
$$


Assim, se $M_{O}>1$, indica uma melhora na produtividade da $\mathrm{DMU}_{\mathrm{i}}$; se $M_{O}<1$, houve uma piora; e, se $M_{\mathrm{O}}=1$, a produtividade manteve-se constante.

$O$ índice de Malmquist permite dividir a variação na produtividade total dos fatores em duas partes: variações na eficiência técnica (ET), ou catch-up, e variações na tecnologia (T), ou frontier-shift, conforme as equações 4 e 5 , respectivamente:

$$
E T=\frac{d_{O}^{t+1}\left(y_{t+1}, x_{t+1}\right)}{d_{O}^{t}\left(y_{t}, x_{t}\right)}
$$

nas quais $d_{O}{ }^{t}\left(y_{t}, x_{t}\right)$ indica a eficiência técnica da $\mathrm{DMU}_{\mathrm{i}}$ no período te $\mathrm{d}_{\mathrm{O}}{ }^{t+1}\left(\mathrm{y}_{t+1}, \mathrm{x}_{t+1}\right)$ mostra a eficiência técnica da $\mathrm{DMU}_{\mathrm{i}}$ no tempo $t+1$.

A equação 4 contabiliza a variação da eficiência técnica relativa entre os dois períodos $t$ e $t+1$, ou seja, capta a mudança da distância em que os fatores de produção observados estão em relação aos fatores de produção mínimos que ainda produzem outputs desejados, que estão sobre a fronteira eficiente, no intervalo de tempo em análise. Se ET > 1, a eficiência técnica melhorou; se ET < 1, a eficiência piorou; e, se ET $=1$, a eficiência técnica se manteve constante.

A equação 5 representa a variação da tecnologia de produção, sendo que essa variação pode ocorrer devido a acréscimos ou decréscimos no percentual de transformação dos inputs em outputs. A interpretação dos valores obtidos para $T$ são similares aos de ET, ou seja, se $T>1$, houve progresso tecnológico, se $T=1$, a tecnologia permaneceu constante e, se $T<1$, houve retrocesso tecnológico ao longo do período de análise.

$$
T=\left[\frac{d_{o}^{t}\left(y_{t+1}, x_{t+1}\right)}{d_{O}^{t+1}\left(y_{t+1}, x_{t+1}\right)} \times \frac{d_{o}^{t}\left(y_{t}, x_{t}\right)}{d_{O}^{t+1}\left(y_{t}, x_{t}\right)}\right]^{1 / 2}
$$

Uma vez que os dados são empilhados, ou seja, dispostos como dados em painel, é possível calcular as medidas de distância necessárias para o índice de Malmquist utilizando-se a técnica de DEA. Assim, para a i-ésima unidade de produção, calculam-se quatro funções de distância para calcular a mudança da produtividade total dos fatores, entre o período $t$ e o período $t+1$. Os problemas de programação linear serão resolvidos admitindo retornos constantes à escala e orientação produto. ${ }^{2}$ Segundo Griefell-Tatjé e Lovell (1995), faz-se necessário considerar retornos constantes à escala, a fim de que as mudanças na produtividade total dos fatores sejam corretamente estimadas.

2 Para mais detalhes a respeito, ver Gomes (2009). 


\subsection{Testes de Convergência}

Segundo Barro e Sala-I-Martin (1991), há dois métodos para se verificar a existência de convergência em uma série temporal: os testes de $\sigma$-convergência e de $\beta$-convergência. $O$ primeiro mede a dispersão de uma série ao longo tempo, o que consiste em verificar se o coeficiente de variação diminui com o decorrer do tempo, isto é, se converge para a média. Porém, as médias de dois períodos distintos não podem ser comparadas quando se trabalha com medidas de eficiência, já que se referem a fronteiras distintas. Dessa forma, considera-se o comportamento do desvio padrão para medir a $\sigma$ - convergência.

Por outro lado, o teste de $\beta$-convergência analisa se há relação negativa entre o valor da série no período inicial e sua taxa de crescimento no segundo período. Assim, esse teste tem como finalidade verificar se os menores indicadores das unidades iniciais aproximam-se da taxa de crescimento das unidades com melhores resultados. Então, quanto mais afastado do estado estacionário, maior será a taxa de crescimento (BARRO; SALA-I-MARTIN, 1991).

O teste de $\beta$-convergência pode ser realizado pelo método dos mínimos quadrados ordinários (MQO), conforme a equação 6:

$$
\frac{1}{T} \ln \left(\frac{y_{i, t}}{y_{i, 0}}\right)=\beta_{1}+\beta_{2} \ln \left(y_{i, 0}\right)+u_{i}
$$

em que $T$ é o intervalo intertemporal entre as séries analisadas; $\mathrm{y}_{\mathrm{i}, 0}$ e $\mathrm{y}_{\mathrm{i}, \mathrm{t}}$ são os períodos inicial e final; $\mathrm{u}_{\mathrm{i}}$ é o termo de erro aleatório; $\beta_{1}$ é o coeficiente de convergência; e $\beta_{2}=-\left(1-e^{-\beta T} / T\right)$ representa a velocidade de convergência. Se $\beta_{2}<0$, pode-se concluir que ocorre $\beta$-convergência no modelo.

Neste trabalho, a relação avaliada está entre a taxa de mudança na eficiência técnica, obtida pela decomposição do índice de Malmquist, e o nível de eficiência inicial, obtido pelo modelo de DEA com retornos variáveis, considerando o output indesejado (emissões de CO2).

\subsection{Fonte e Tratamento dos Dados}

As variáveis utilizadas neste estudo são: população (em milhões de habitantes), consumo primário total de energia (quilotones de petróleo equivalente), PIB (em dólares correntes) e emissões de $\mathrm{CO} 2$ (em milhões de toneladas métricas). O uso de tais variáveis se justifica pelo fato de influenciarem as emissões de GEE e também a formação do PIB. Além disso, essas variáveis são comumente utilizadas em trabalhos aplicados quando se leva em consideração outputs indesejados, 
como nos trabalhos de Gomes e Lins (2007) e Zhang et al. (2011). As informações a respeito do PIB, população e uso total de energia foram extraídas do Banco Mundial e, a respeito das emissões de $\mathrm{CO} 2$, do Energy Information Administration (EIA).

O modelo proposto neste estudo sugere como outputs o PIB e as emissões de $\mathrm{CO} 2$ e como inputs a população e o consumo total de energia. A emissão de $\mathrm{CO} 2$ é um output indesejado do processo produtivo (GOMES, 2003), logo é o produto que deve ter sua produção minimizada.

$\mathrm{Na}$ literatura encontram-se algumas formas alternativas de tratamento para outputs indesejados, sendo a escolha arbitrária. Por exemplo, Lovell et al. (1995) utilizam o inverso do output indesejado como output. No entanto, esse método para o presente trabalho não seria a melhor alternativa, uma vez que há países com altos valores de emissões de $\mathrm{CO} 2 \mathrm{e}$, ao fazer o seu inverso, retornar-se-ia a um valor muito pequeno, o que poderia tornar o resultado incoerente.

Outra forma de lidar com outputs indesejados é o proposto por Rheinhard, Lovell e Thijssen (1999), em que o output indesejado passa a ser tratado no modelo de DEA como input. A incorporação de outputs indesejados como inputs gera o mesmo conjunto de possibilidades de produção que incorporar os outputs como desejáveis. Segundo Gomes (2003), essa abordagem é mais intuitiva e representa melhor a lógica causal do modelo proposto ao minimizar o uso dos insumos (inputs).

As DMU do modelo de DEA são os países do Anexo I e do Não Anexo I do Protocolo de Quioto, totalizando 186 países. Entretanto, para 67 países não existem informações disponíveis sobre alguma variável, portanto foram retirados da amostra, restando, assim, 121 países para este estudo.

\section{Análise dos Resultados}

\subsection{Análise da Decomposição do Índice de Malmquist}

O Protocolo de Quioto tem como objetivo reduzir as emissões de GEE em pelo menos 5\% em relação aos níveis de 1990 até o período entre 2008 e 2012 (BRASIL, 1997). Para isso, espera-se que haja mudança tecnológica e na eficiência do sistema produtivo dos países para que tal objetivo seja alcançado. Para verificar essas mudanças, utilizou-se o índice de Malmquist, que revela alterações da produtividade e sua decomposição mostra mudanças tecnológicas e de eficiência. A escolha do ano inicial, 1995, deve-se ao fato de o Protocolo de Quioto ter sido realizado em 1997. Dessa forma, o ano inicial serve como um período neutro, em que não havia compromissos formais quanto à mitigação das emissões, permitindo, assim, verificar possíveis alterações do sistema produtivo influenciadas pelo protocolo. O último ano, 2010, deve-se ao fato de não haver dados disponíveis para todos os países analisados para anos mais recentes. 
Neste estudo, os inputs são a população, as emissões de $\mathrm{CO} 2$ (output indesejável) e o consumo de energia primária, e o output é o PIB. A Tabela 1 apresenta a análise descritiva dos resultados dos períodos em estudo para o índice de Malmquist (PTF) e sua decomposição.

Tabela 1 - Resumo estatístico-descritivo dos resultados para os períodos em análise

\begin{tabular}{|c|c|c|c|c|c|c|}
\hline Ano & Variável & Média & Mediana & $\begin{array}{l}\text { Desvio } \\
\text { padrão }\end{array}$ & Mínimo & Máximo \\
\hline \multirow{3}{*}{$1995-2000$} & $\begin{array}{l}\text { Mudança de } \\
\text { eficiência } \\
\text { técnica }\end{array}$ & 1,3515 & 1,2730 & 0,4103 & 0,570 & 2,644 \\
\hline & $\begin{array}{l}\text { Mudança } \\
\text { tecnológica }\end{array}$ & 0,7781 & 0,7640 & 0,0378 & 0,760 & 0,921 \\
\hline & $\begin{array}{l}\text { Mudança na } \\
\text { PTF }\end{array}$ & 1,0510 & 0,9770 & 0,3179 & 0,438 & 2,029 \\
\hline \multirow{3}{*}{$2000-2005$} & $\begin{array}{l}\text { Mudança de } \\
\text { eficiência } \\
\text { técnica }\end{array}$ & 1,0325 & 0,9950 & 0,3895 & 0,402 & 3,891 \\
\hline & $\begin{array}{l}\text { Mudança } \\
\text { tecnológica }\end{array}$ & 1,4900 & 1,4600 & 0,0717 & 1,449 & 1,743 \\
\hline & $\begin{array}{l}\text { Mudança na } \\
\text { PTF }\end{array}$ & 1,5349 & 1,4750 & 0,5690 & 0,582 & 5,722 \\
\hline \multirow{3}{*}{$2005-2010$} & $\begin{array}{l}\text { Mudança de } \\
\text { eficiência } \\
\text { técnica }\end{array}$ & 1,0900 & 0,9910 & 0,3543 & 0,482 & 3,288 \\
\hline & $\begin{array}{l}\text { Mudança } \\
\text { tecnológica }\end{array}$ & 1,4289 & 1,4210 & 0,0765 & 1,274 & 1,563 \\
\hline & $\begin{array}{l}\text { Mudança na } \\
\text { PTF }\end{array}$ & 1,5607 & 1,4080 & 0,5286 & 0,729 & 4,672 \\
\hline \multirow{3}{*}{$1995-2010$} & $\begin{array}{l}\text { Mudança de } \\
\text { eficiência } \\
\text { técnica }\end{array}$ & 1,1191 & 1,0520 & 0,2251 & 0,684 & 2,285 \\
\hline & $\begin{array}{l}\text { Mudança } \\
\text { tecnológica }\end{array}$ & 1,1822 & 1,1630 & 0,0298 & 1,151 & 1,282 \\
\hline & $\begin{array}{l}\text { Mudança na } \\
\text { PTF }\end{array}$ & 1,3211 & 1,2430 & 0,2587 & 0,831 & 2,653 \\
\hline
\end{tabular}

Fonte: Elaboração própria a partir dos resultados da pesquisa.

Ao se analisar a Tabela 1, e relembrando que, para haver mudança no índice de Malmquist, e/ou em qualquer um de seus componentes, os resultados devem ser maiores que um, para uma alteração positiva, e menores que um, para uma 
mudança negativa. Assim, pode-se perceber que houve retrocesso tecnológico em todos os países no período de elaboração do Protocolo de Quioto (1995-2000). Isso indica que no período de formulação do protocolo e no período de assinaturas (que ocorreu em 1998) nenhum país demonstrou compromissos concretos quanto à mitigação de GEE. Por outro lado, observa-se que, entre 1995 e 2000, houve mudanças tanto positivas quanto negativas na eficiência técnica e na PTF. Para os 121 países analisados, nesse período, 48\% apresentaram melhora na PTF e $52 \%$, piora. Com relação à eficiência técnica, $82 \%$ dos países obtiveram melhora, $16 \%$, piora e $2 \%$ permaneceram constantes.

Analisando ainda a Tabela 1, pode-se perceber que o progresso tecnológico ocorreu, de fato, entre 2000 e 2005. Destaca-se que, em 2005, o Protocolo de Quioto foi ratificado. Nesse período, todos os países em análise tiveram progresso tecnológico, e o mesmo ocorreu entre 2005 e 2010, período que abrange a ocasião em que os países do Anexo I deveriam tomar atitudes quanto à mitigação de GEE. Nota-se que há um esforço conjunto entre os países para que reduzam, concretamente, as emissões de GEE, conforme o proposto pelo protocolo, uma vez que, além do progresso tecnológico, houve também mudança positiva da eficiência técnica.

Com relação a todo o período analisado, os resultados para o índice de Malmquist mostram que, para os 121 países em estudo, houve um aumento médio, entre 1995 e 2010, de 32,11\% na produtividade. Como a produtividade é um índice, os valores médios anuais são médias geométricas, ou seja, é a raiz décima quinta do produto das taxas de crescimento para os anos analisados. Isso significa que, para os 121 países, houve um aumento médio anual de 1,87\% na produtividade. O Azerbaijão obteve o maior ganho de produtividade no período, 165,3\%, enquanto que Benim teve o pior resultado, com perda de 16,9\% (ver Tabela 1).

Com relação aos resultados da decomposição do índice de Malmquist, embora a eficiência média tenha aumentado $11,91 \%$, a mudança tecnológica foi a principal fonte da melhora da PTF (18,22\%). O progresso técnico foi verificado em todos os países em estudo, o que corrobora sua importância na PTF. O país que apresentou a maior variação tecnológica foi os Emirados Árabes Unidos (28,2\%) e, a menor, foram o Cazaquistão e a Eslovênia (15,1\%). A Figura 1 mostra as mudanças tecnológicas ocorridas nos países em estudo. 
Figura 1 - Variação tecnológica dos 121 países em estudo, entre 1995 e 2010

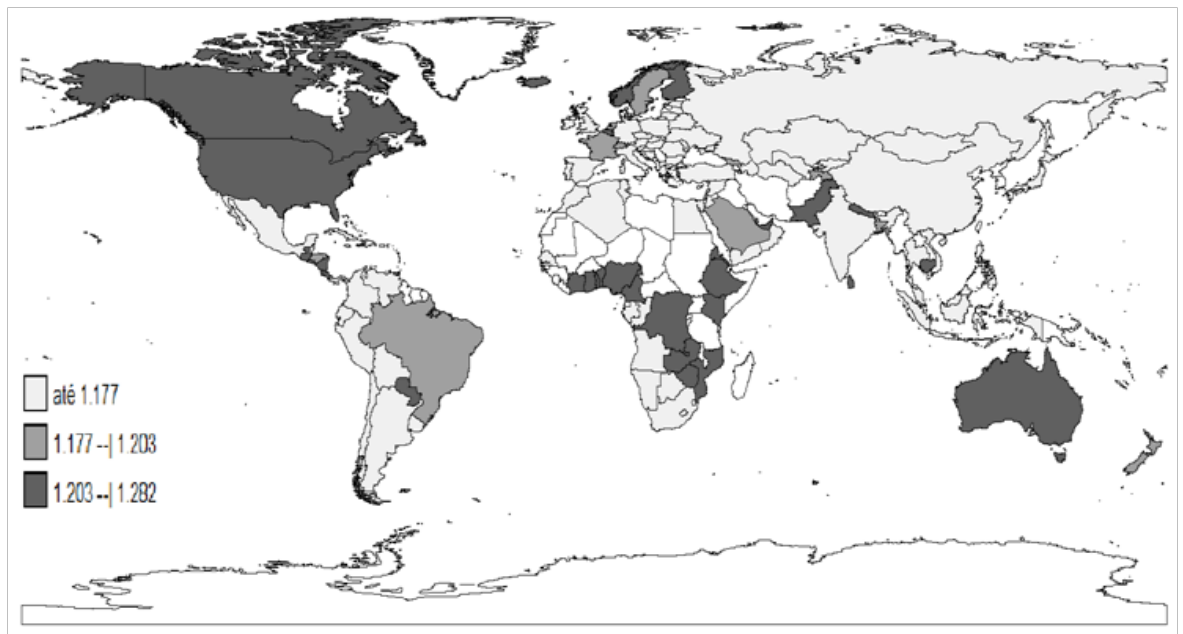

Fonte: Elaboração própria a partir dos resultados da pesquisa.

Nota: Países em branco não fazem parte da amostra.

Como pode ser observado na Figura 1, a maior variação tecnológica está concentrada na América do Norte, enquanto que as menores se concentram na América do Sul, Ásia, Europa e extremo sul da África. Um resultado interessante é que a maior parte dos países do Anexo I está entre os países que obtiveram a menor variação tecnológica positiva, ou seja, progresso técnico.

Apesar da constatação do progresso tecnológico, a maioria dos países não é eficiente tecnicamente (ver Figura 2). Apenas 11 países são 100\% eficientes, ${ }^{3}$ sendo oito do Anexo I: Estados Unidos, França, Islândia, Japão, Luxemburgo, Malta, Noruega e Suíça. A eficiência média é de $42 \%$, sendo que a média para os países do Anexo I é de 61\% e dos países do Não Anexo I é de 32\%. Dessa forma, apesar do menor progresso tecnológico verificado entre os países do Anexo I, esses são mais eficientes tecnicamente ao considerar as emissões de $\mathrm{CO} 2$, indicando o seu maior compromisso com as mudanças climáticas. Cabe destacar a presença dos Estados Unidos, o único país do Anexo I que não ratificou o Protocolo de Quioto, entre os mais eficientes na formação do PIB quando se leva em conta emissões de $\mathrm{CO} 2$. Entretanto, pelos resultados, apesar da não adesão ao protocolo, parece que o país adotou medidas de mitigação das emissões de GEE voluntariamente, pois, além de estar entre os mais eficientes, encontra-se entre os países que obtiveram maior progresso tecnológico.

3 Eritreia, Estados Unidos, França, Gabão, Islândia, Japão, Luxemburgo, Malta, Namíbia, Noruega e Suíça. 
Figura 2 - Eficiência técnica dos 121 países em estudo no ano de 2010

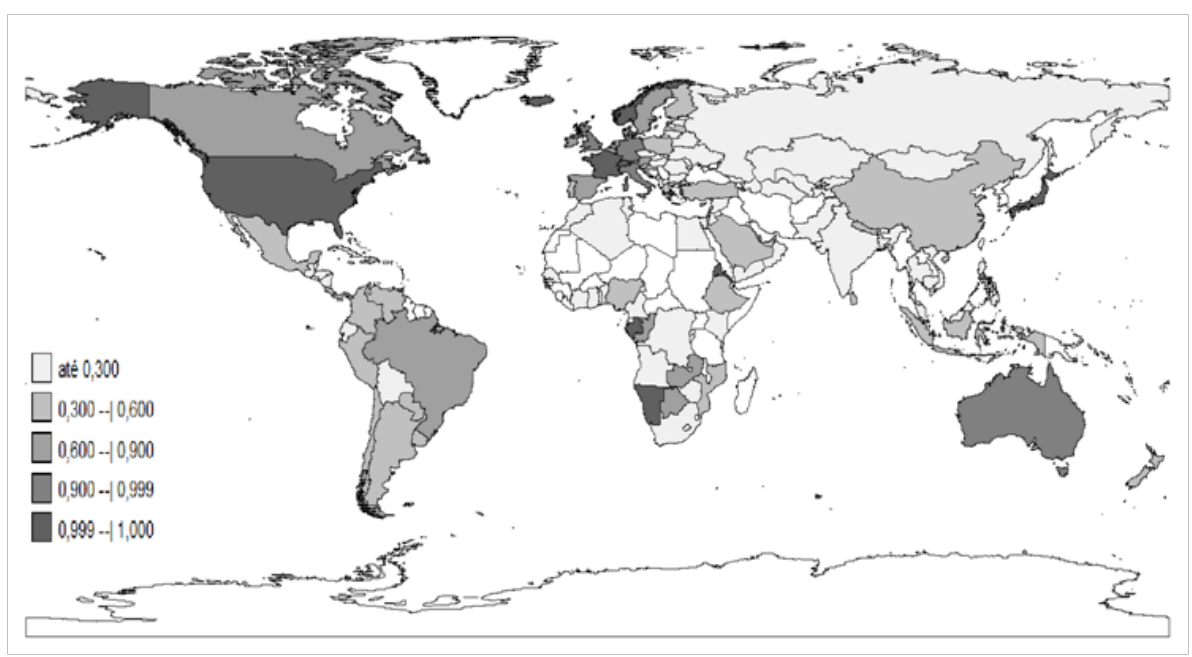

Fonte: Elaboração própria a partir dos resultados da pesquisa.

Nota: Países em branco não fazem parte da amostra.

Como destacam Lutsey e Sperling (2008), nos Estados Unidos, cada vez mais os governos a níveis subnacionais estabelecem metas de emissão e planos de implantação de redução de GEE. Enquanto o governo federal concentra-se em programas de mitigação voluntários, os governos locais, regionais e estaduais têm intensificado ações concretas de mitigação de suas emissões, estabelecendo metas de redução de emissões semelhantes aos do Protocolo de Quioto. Por conseguinte, à medida que mais esforços de mitigação se concretizam, reduções significativas de emissões em todo país são percebidas. Isso indica que os Estados Unidos são mais comprometidos com a mitigação das mudanças climáticas do que geralmente são reconhecidos.

No setor de energia elétrica, por exemplo, existe um conjunto de estratégias dos entes federativos americanos para diminuir as emissões de GEE. Embora as estratégias sejam diferentes entre os estados, têm se mostrado eficazes. Os estados de Massachusetts e New Hampshire, por exemplo, implementaram políticas de redução obrigatórias de plantas mais antigas, enquanto que os estados de Oregan e Washington regulamentaram os níveis de emissões de novas usinas (RAMSEUR, 2007).

Destaca-se, ainda, que três países africanos (Eritreia, Gabão e Namíbia), países fora do Anexo I, obtiveram 100\% de eficiência (ver Figura 2). Tais países possuem forte vocação agrícola. Na Eritreia, por exemplo, 80\% da população está envolvida com a agricultura de subsistência. Com relação às economias do Gabão e da Namíbia, além da agricultura, destaca-se a exportação de petróleo e minerais. Como grande parte do petróleo é refinada e consumida em outros países, e dadas 
as características de subsistência com as quais vive grande parte da população dessas nações, os inputs se ajustam melhor à fronteira para produzir um PIB relativamente muito pequeno, tornando-os eficientes.

\subsection{Testes de Convergência para as Medidas de Eficiência}

\subsubsection{Teste de $\sigma$-Convergência}

O primeiro instrumento para se identificar uma provável convergência das medidas de eficiência da produção dos países em estudo é o teste de $\sigma$-convergência. Esse teste consiste, simplesmente, em analisar o que está ocorrendo com as medidas de eficiência estimadas separadamente em dois períodos de tempo. Os resultados para os países em análise encontram-se na Tabela 2.

Tabela 2 - Desvios padrão das medidas de eficiência em 1995 e 2010 ( $\sigma$-convergência)

\begin{tabular}{lccc}
\hline \multirow{2}{*}{ Região } & \multicolumn{2}{c}{ Desvio padrão } & \multirow{2}{*}{ Variação } \\
\cline { 2 - 3 } & $\mathbf{1 9 9 5}$ & $\mathbf{2 0 1 0}$ & \\
\hline Anexo I & 0,3283 & 0,3190 & $-2,83 \%$ \\
Não Anexo I & 0,2211 & 0,2088 & $-5,56 \%$ \\
Total & 0,2747 & 0,2639 & $-3,93 \%$ \\
\hline
\end{tabular}

Fonte: Elaboração própria a partir dos resultados da pesquisa.

Os resultados apresentados na Tabela 2 permitem averiguar que, tanto para os países do Anexo I, quanto para os países do Não Anexo I, houve redução no desvio padrão das medidas de eficiência. Em média, o desvio padrão dessas medidas diminuiu 3,93\%, com maior redução para os países fora do Anexo I (5,56\%). É importante salientar que as medidas de eficiência foram calculadas isoladamente nos dois períodos, ou seja, foi estimada uma fronteira de eficiência para o ano de 1995 e outra para 2010. Isso implica que as medidas de eficiência calculadas para uma região específica não podem ser comparadas, uma vez que se referem a fronteiras diferentes. Contudo, a dispersão das medidas pode ser comparada, isto é, como se encontram dispersas as medidas em relação à fronteira de determinado período, relativamente a outro período.

$\mathrm{O}$ teste de $\sigma$-convergência demonstra apenas que a dispersão das medidas de eficiência em relação às fronteiras dos dois períodos está reduzindo. No entanto, não fornece evidências concretas de que está havendo convergência entre os países para um patamar superior de eficiência. Para isso, é necessário comparar os valores iniciais com as taxas de mudança na eficiência, o que pode ser feito pelo teste de $\beta$-convergência. 


\subsubsection{Teste de $\beta$-Convergência}

O teste de $\beta$-convergência verifica se há relação negativa entre o nível de eficiência original e a taxa de mudança na eficiência (equação 6). Isso significa que, para haver convergência, é necessário que os países que apresentaram menores valores de eficiência no período inicial obtenham maiores taxas de mudança na eficiência. Primeiramente, o teste de $\beta$-convergência foi realizado para todos os países em análise, conforme os resultados apresentados na Tabela 3. A forma funcional escolhida foi a duplo-log, uma vez que o coeficiente estimado fornece diretamente o valor da elasticidade. ${ }^{4}$

Tabela 3 - Coeficientes estimados para o teste de $\beta$-convergência ${ }^{5}$

\begin{tabular}{lcccc}
\hline \multicolumn{1}{c}{ Variável } & $\begin{array}{c}\text { Coeficiente } \\
\text { estimado }\end{array}$ & Erro padrão & Estatística $\boldsymbol{t}$ & Valor-p \\
\hline Intercepto & $-0,0928$ & 0,0253 & $-4,2622$ & 0,0004 \\
ln eficiência de 1995 & $-0,1294$ & 0,0163 & $-7,9034$ & 0,0000 \\
\hline Observações $=121$ & $\mathrm{R}^{2}=0,4735$ & & & \\
\hline
\end{tabular}

Fonte: Elaboração própria a partir dos resultados da pesquisa.

Os resultados apresentados na Tabela 3 revelam que o coeficiente estimado para a medida de eficiência para o período inicial (1995) é estatisticamente significativo e o sinal coeso ao esperado. Em outras palavras, pode-se inferir que menores valores iniciais de eficiência estão associados a maiores taxas de mudança, cuja elasticidade foi de $-0,1294$.

É importante destacar que neste estudo foram levadas em consideração para o cálculo dos valores das eficiências as emissões de CO2. De acordo com os resultados, a hipótese de $\beta$-convergência foi confirmada, então isso significa que está havendo maior preocupação dos países quanto à mitigação desses gases por meio de uma melhor eficiência no processo produtivo.

A Figura 3 permite ver a relação negativa entre o nível de eficiência no período inicial e o índice de mudança na eficiência. Pode-se verificar que a maior parte dos países que atingiram eficiência máxima em 1995 não apresentou ganho relativo de eficiência. Por outro lado, as menores eficiências estimadas em 1995 estão associadas às maiores taxas de mudança na eficiência, o que evidencia a existência de $\beta$-convergência.

$4 \quad$ Para mais detalhes, ver Gujarati (2011).

5 Foi detectado indício de heterocedasticidade pelo teste de White. Dessa forma, a violação do pressuposto de homocedasticidade foi corrigida por meio dos erros padrões robustos de White. Para mais detalhes, ver Gujarati (2011). O mesmo teste e correção foram realizados na regressão apresentada na Tabela 4. 
Figura 3 - Dispersão entre nível de eficiência técnica inicial e índice de mudança na eficiência e linha de tendência ajustada

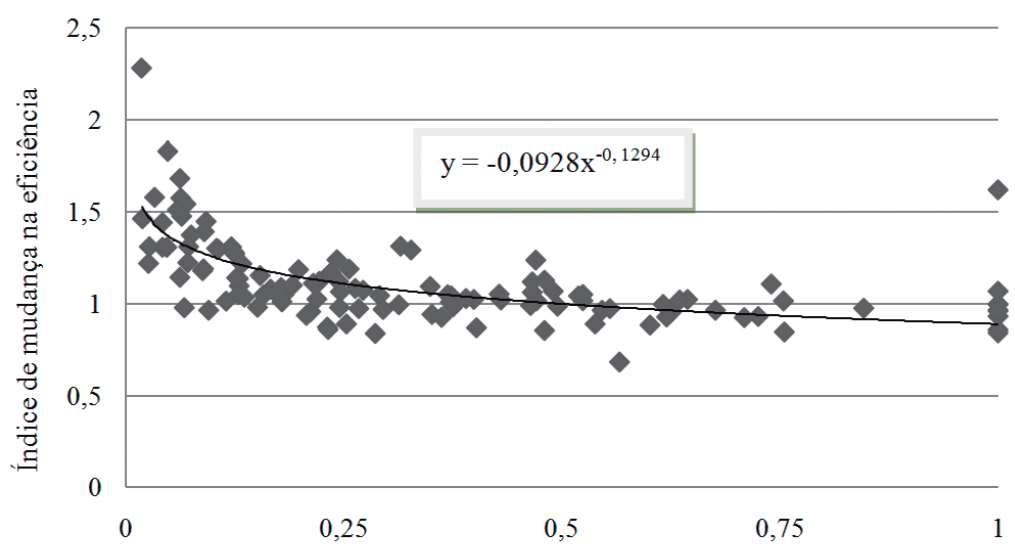

Efíciência de 1995

Fonte: Elaboração própria a partir dos resultados da pesquisa.

O teste de $\beta$-convergência também foi realizado, separadamente, para os países que compõem o Anexo I do Protocolo de Quioto e também para países que estão fora do Anexo I, por meio de uma variável dummy. O objetivo dessa divisão é verificar se há diferença no processo de convergência em cada região. Os resultados da estimativa encontram-se na Tabela 4, e os coeficientes exibidos referem-se ao valor final da elasticidade, ou seja, já foi acrescida a influência captada pela variável dummy.

Tabela 4 - Coeficientes estimados para o teste de $\beta$-convergência para os países do Anexo 1 e do Não Anexo 1

\begin{tabular}{lcccc}
\hline \multicolumn{1}{c}{ Variável } & $\begin{array}{c}\text { Coeficiente } \\
\text { estimado* }\end{array}$ & Erro padrão & Estatística $\boldsymbol{t}$ & Valor-p \\
\hline Anexo 1 & $-0,0479$ & 0,0213 & 4,0653 & 0,0001 \\
Não Anexo 1 & $-0,1343$ & 0,0321 & $-4,1798$ & 0,0001 \\
\hline Observações = 121 & $\mathrm{R} 2=0,522$ & & & \\
\hline
\end{tabular}

Fonte: Elaboração própria a partir dos resultados da pesquisa.

Nota: *Coeficientes finais já adicionados ao valor da variável dummy. 
Para ambos os grupos de países, os coeficientes estimados apresentaramse estatisticamente significativos e com sinais negativos, ou seja, houve processo de convergência nas medidas de eficiência técnica. Todavia, a maior velocidade de convergência ocorreu no grupo de países que não possui obrigações formais quanto à mitigação de GEE e também com a menor eficiência média verificada (Não Anexo I). Em outras palavras, pode-se inferir que, apesar de não haver uma obrigação intrínseca quanto à mitigação no grupo de países do Não Anexo I, compromissos voluntários estão sendo adotados para minimizar o processo de aquecimento global. Tal fato reforça a conclusão de convergência do sistema produtivo mundial, aliada a práticas produtivas mais sustentáveis nas regiões menos eficientes.

\section{Considerações Finais}

O processo de construção de um produto pode resultar em subprodutos muitas vezes inevitáveis e também indesejados. Esse resultado ocorre na formação do PIB das nações. O processo de formação do PIB, além de contribuir para as rendas dos indivíduos, também resulta em emissões de GEE, responsáveis pelas mudanças climáticas. A DEA é uma ferramenta capaz de lidar com outputs indesejados, e pelo índice de Malmquist é possível verificar se há progresso tecnológico e mudança na eficiência que poderiam reduzir os níveis de emissões de GEE conforme o planejado no Protocolo de Quioto.

As metas de redução para os países do Anexo I deveriam ser cumpridas no período entre 2008 e 2012. De fato, neste trabalho, foi constatado progresso tecnológico em todos os países analisados, o que indica uma preocupação das nações quanto às mudanças climáticas. Entretanto, a grande maioria dos países não é eficiente tecnicamente, o que leva a crer que, apesar do progresso tecnológico, ainda é necessário haver mais esforço e cooperação entre os países para que seus processos produtivos sejam mais eficientes, minimizando as emissões de GEE.

Os resultados deste trabalho mostram que, em média, os países do Anexo I são mais eficientes que os países do Não Anexo I, o que não é um fato surpreendente, uma vez que os primeiros possuem obrigações formais quanto à mitigação de GEE. No entanto, o fato de diferentes grupos de países apresentarem valores distintos e, às vezes, muito discrepantes em determinado fator conduz à hipótese de convergência. Esta tem sido tema recorrente nas pesquisas acadêmicas, e as análises que em princípio eram restritas apenas à convergência de renda entre os países expandiram-se para outros fatores, níveis espaciais e setores.

Neste estudo, foi testada a hipótese de convergência na eficiência do sistema produtivo dos países ao considerar as emissões de $\mathrm{CO} 2$, o que foi confirmada pelos testes de $\sigma$-convergência e $\beta$-convergência. Assim, pode-se dizer que menores 
valores iniciais de eficiência estão associados a maiores taxas de crescimento da eficiência, o que indica maior preocupação mundial quanto a práticas produtivas mitigadoras de GEE.

A redução do desvio padrão ( $\sigma$-convergência) e a relação negativa entre o valor inicial da eficiência e a taxa de mudança ( $\beta$-convergência) indicam que as regiões mais ineficientes estão melhorando, o que caracteriza maior homogeneidade na eficiência do sistema produtivo mundial. Quando a análise foi feita separadamente, verificou-se que nos países do Não Anexo I, países menos eficientes, o processo de convergência foi mais intenso. Dessa forma, no longo prazo, apesar desses países não terem obrigações formais quanto à mitigação de GEE, tendem a alcançar os países mais desenvolvidos tecnicamente, o que contribuiria para minimizar os efeitos das mudanças climáticas.

\section{Referências}

BANG, G. et al. The United States and international climate cooperation: international "pull" versus domestic "push". Energy Policy, v. 35, n. 2, p. 1282-1291, 2007.

BANKER, R. D.; CHARNES, H.; COOPER, W. W. Some models for estimating technical and scale inefficiencies in data envelopment analysis. Management Science, v. 30, n. 9, p. 10781092, 1984.

BARRO, R. J.; SALA-I-MARTIN, X. Convergence across states and regions. Brookings Papers on Economic Activity, v. 22, n. 1, p. 107-182, 1991.

BRÄNNLUND, R.; LUNDGREN, T. Environmental policy without costs? A review of the porter hypothesis. International Review of Environmental and Resource Economics, v. 3, n. 2, p. 75-117, 2009.

BRASIL. Ministério da Ciência e Tecnologia. Protocolo de Quioto. Brasília, DF: MCT, 1997. Disponível em: <http://mudancasclimaticas.cptec.inpe.br/ rmclima/pdfs/Protocolo_ Quioto.pdf>. Acesso em: 29 mar. 2013.

CAVES, D. W.; CHRISTENSEN, L. R.; DIEWERT, W. E. The economic theory of index numbers and the measurement of input, output and productivity. Econometrica, v. 50, n. 6, p. 1393-1414, 1982.

CHARNES, A. et al. Data envelopment analysis: theory, methodology and application. Dordrecht: Kluwer Academic, 1994.

CHARNES, A.; COOPER, W. W.; RHODES, E. Measuring the efficiency of decision making units. European Journal of Operational Research, v. 2, n. 6, p. 429-444, 1978.

COELLI, T. J. et al. An introduction to efficiency and productivity analysis. 2. ed. New York: Springer, 2005.

DALY, H. E. Georgescu-Roegen versus Solow/Stiglitz. Ecological Economics, v. 22, n. 3, p. 261-266, 1997. 
DALY, H. E.; FARLEY, J. Ecological economics: principles and applications. Washington, D.C.: Island Press, 2010.

DE LA TORRE, A.; FAJNZYLBER, P.; NASH, J. D. Low carbon, high growth: Latin American responses to climate change-an overview. Washington, D.C.: World Bank Publications, 2009.

DOMAR, E. D. Capital expansion, rate of growth, and employment. Econometrica, v. 14, n. 2, p. 137-147, 1946.

EDENHOFER, O.; BAUER, N.; KRIEGLER, E. The impact of technological change on climate protection and welfare: insights from the model MIND. Ecological Economics, v. 54, n. 2, p. 277-292, 2005.

FÄRE, R. et al. Productivity growth, technical progress, and efficiency change in industrialized countries. American Economic Review, v. 84, n. 1, p. 66-83, Mar. 1994.

FEIJÓ, F. T.; AZEVEDO, A. F. Z. Comércio e meio ambiente: políticas ambientais e competitividade no âmbito da ALCA. Economia Aplicada, v. 10, n. 4, p. 561-587, 2006.

FERREIRA, C. M. C.; GOMES, A. P. Análise envoltória de dados: teoria, modelos e aplicações. Viçosa: Editora da UFV, 2012.

FISCHER, C.; NEWELL, R. G. Environmental and technology policies for climate mitigation. Journal of Environmental Economics and Management, v. 55, n. 2, p. 142-162, 2008.

GEORGESCU-ROEGEN, N. The entropy law and the economic process in retrospect. Eastern Economic Journal, v. 12, n. 1, p. 3-25, 1986.

GOMES, E. G. Modelos de análise de envoltória de dados com ganhos de soma zero. 2003. Tese (Doutorado em Engenharia de Produção) - Instituto Alberto Luiz Coimbra de PósGraduação e Pesquisa de Engenharia, Universidade Federal do Rio de Janeiro, Rio de Janeiro, 2003.

GOMES, E. G.; LINS, M. P E. Modelling undesirable outputs with zero sum gains data envelopment analysis models. Journal of the Operational Research Society, v. 59, n. 5, p. 616623, 2007.

GRIFFELL-TATJÉ, E.; LOVELL, C. A. K. Anote on the Malmquist productivity index. Economics Letters, v. 47, n. 2. p. 169-175, 1995.

GUJARATI, D. N; PORTER, D. C. Econometria básica. Porto Alegre: AMGH, 2011.

HARROD, R. F. An essay in dynamic theory. The Economic Journal, v. 49, n. 193, p. 14-33, 1939.

INTERGOVERNMENTAL PANEL ON CLIMATE CHANGE. Climate change 2013: the physical science basis. Working group I contribution to the IPCC fifth assessment report, summary for policymakers. 2013. Disponível em: < http:/www.climatechange2013.org/images/uploads/ WGIAR5-SPM_Approved27Sep2013.pdf>. Acesso em: 09 out. 2013.

INTERNATIONAL ENERGY ADMINISTRATION. Worldwide trends in energy use and effciency: key insights from IEA indicator analysis. Paris: International Energy Agency, 2008. 
JAFFE, A. B. et al. Environmental regulation and the competitiveness of US manufacturing: what does the evidence tell us? Journal of Economic Literature, v. 33, n. 1, p. 132-163, 1995.

LINS, M. P. E.; MEZA, L. A. Análise envoltória de dados e perspectivas de integração no ambiente de apoio à tomada de decisão. Rio de Janeiro: COPPE/UFRJ, 2000.

LOVELL, C. A. K. et al. Measuring macroeconomic performance in the OECD: a comparison of European and non-European countries. European Journal of Operational Research, v. 87, n. 3, p. 507-518, 1995.

LUTSEY, N.; SPERLING, D. America's bottom-up climate change mitigation policy. Energy Policy, v. 36, n. 2, p. 673-685, 2008.

MILLS, E. Building commissioning: a golden opportunity for reducing energy costs and greenhouse gas emissions. Berkeley: Lawrence Berkeley National Laboratory, 2009.

MURTY, S.; RUSSELL, R. R.; LEVKOFF, S. B. On modeling pollution-generating technologies. Journal of Environmental Economics and Management, v. 64, n. 1, p. 117-135, 2012.

NEUMAYER, E. Weak versus strong sustainability: exploring the limits of two opposing paradigms. Cheltenham: Edward Elgar Publishing, 2003.

RAMSEUR, J. L. Climate change: action by states to address greenhouse gas emissions. Washington, D.C.: Congressional Research Service, 2007.

RHEINHARD, S.; LOVELL, C. A. K.; THIJSSEN, G. Econometric estimation of technical and environmental efficiency: an application to Dutch dairy farms. American Journal of Agricultural Economics, v. 81, n. 1, p. 44-60, 1999.

ROMER, D. Advanced macroeconomics. 2. ed. New York: McGraw-Hill, 2006.

SILVEIRA, J. Q.; MEZA, L. A.; MELLO, J. C. C. B. S. Identificação de Benchmarks e antiBenchmarks para companhias aéreas usando modelos DEA e fronteira invertida. Produção, v. 22, n. 4, p. 788-795, set.-dez. 2012.

SOLOW, R. M. A contribution to the theory of economic growth. The Quarterly Journal of Economics, v. 70, n. 1, p. 65-94, 1956.

. On the intergenerational allocation of natural resources. The Scandinavian Journal of Economics, v. 88, n. 1, p. 141-149, 1986.

. Technical change and the aggregate production function. The Review of Economics and Statistics, v. 39, n. 3, p. 312-320, 1957.

The economics of resources or the resources of economics. The American Economic Review, v. 64, n. 2, p. 1-14. 1974.

UNITED NATIONS. Framework Convention on Climate Change. List of Annex: Parties to the Convention. 2013. Disponível em: <http://unfccc.int/parties_and_observers/parties/annex_i/ items/ 2774.php >. Acesso em: 30 jun. 2013. 
ZHANG, C. et al. Productivity growth and environmental regulations-accounting for undesirable outputs: Analysis of China's thirty provincial regions using the MalmquistLuenberger index. Ecological Economics, v. 70, n. 12, p. 2369-2379, 2011.

Recebido em: 26/11/2013. Aceito em: 11/11/2014. 